\title{
Misfit Dislocations Study in MOVPE Grown Lattice-Mismatched InGaAs/GaAs Heterostructures by Means of DLTS Technique
}

\author{
L. GelczuK*, M. DA̧Browska-Szata, G. Jóźwiak \\ AND D. RADZIEWICZ
}

Faculty of Microsystem Electronics and Photonics

Wrocław University of Technology

Janiszewskiego 11/17, 50-372 Wrocław, Poland

\begin{abstract}
Two deep traps associated with lattice-mismatch induced defects in n-type $\operatorname{In}_{0.042} \mathrm{Ga}_{0.958} \mathrm{As} / \mathrm{GaAs}$ heterostructures and three deep point traps were observed by means of DLTS method. In order to determine the overlapping DLTS-line peaks parameters precisely, high resolution Laplace DLTS studies werw performed. A simple procedure of distinguishing between point and extended defects in DLTS measurements was used.
\end{abstract}

PACS numbers: 71.55.-i, 71.55.Eq, 71.20.Nr

\section{Introduction}

The $\operatorname{In}_{x} \mathrm{Ga}_{1-x}$ As/GaAs ternary compound systems are of considerable interest among lattice-mismatched heterostructures due to their potential applications in high-speed electronic and optoelectronic devices. The epitaxial growth of these heterostructures is accompanied by strain in the epitaxial layer resulting from the differences in lattice parameters between the substrate and the epilayer. The lattice mismatch leads to the formation of misfit dislocations, typical representatives of extended defects. Misfit dislocations are observed when the thickness of the overlayer exceeds strain-dependent critical layer thickness and they are accompanied by threading dislocations which propagate into the epitaxial layer crossing the whole thickness of the structure. It is particularly detrimental to reliability

*corresponding author; e-mail: e- mail: Lukasz.Gelczuk@pwr.wroc.pl 
and performance of devices. Both kinds of dislocations can give rise to energy level in the band gap acting as recombination centers or traps for free carriers [1].

Deep level transient spectroscopy (DLTS) is an extensively used diagnostic method for studying deep level defects in semiconductors. It provides important defect characteristics such as energy level position in the band gap, capture cross-section, and defect concentration [2]. Although the DLTS technique has been preliminarily developed for characterizing simple point defects, it can be also successfully applied for studying extended defects such as dislocations [3]. A quantitative improvement in DLTS resolution was reached by using the Laplace transform, the method for the emission rate analysis. The resolution of method was increased by more than an order of magnitude [4].

In this paper we report results of our investigations of deep levels properties being a result of the misfit and threading dislocations as well as preexisting simple point defects in $\mathrm{In}_{0.042} \mathrm{Ga}_{0.958} \mathrm{As} / \mathrm{GaAs}$ heterostructures. The combination of the lock-in and Laplace DLTS (LDLTS) methods has been successfully applied for studying these two kinds of defects. Moreover, a procedure of distinguishing between point and extended defects in DLTS measurements has been used. It made possible the determination of the origin of these defects and their identification.

\section{Analysis of DLTS measurements in case of extended defects}

Deep level transient spectroscopy has been developed for characterizing simple point defect but a similar application for extended defects is still an open question [3]. It is due to the fact that for extended defects (e.g. dislocations) DLTS data cannot be interpreted unambiguously [5]. According to the authors [6-8], the DLTS lines usually show characteristic symmetric or asymmetric broadening connected with a non- exponential transient of electron emission from the traps. For example, Kimerling and Patel [6] found asymmetrically broadened DLTS lines associated with dislocations in $\mathrm{Si}$, which survived at high temperatures. Anomalous symmetrical line broadening was also studied by Omling et al. [7, 8]. They observed a deep electron trap in GaAs1-xPx ternary compound caused by so-called alloy broadening and after plastic deformation of Si. The latter was attributed to the effect of deformation- induced disorder on the energy level and capture crosssection of point defects related to dislocations. Unfortunately, the DLTS spectrum can be also deformed by a large trap concentration [9], capture from free-carrier tails in a depletion region [10], an inhomogeneous carrier concentration depth profile [11], as well as strain fields and defect interaction [12]. Therefore the frequently observed DLTS line broadening can be attributed to the existence of dislocations, however, other sources of the effect are possible.

It is well known that the principal argument for the assignment of the dislocations-related traps is a logarithmic capture kinetics for majority charge carriers $[8,12,13]$. It results from the repulsive Coulomb interaction between a 
free charge carrier just being captured and other charges captured at the dislocation line [12]. In DLTS measurements, it manifests itself as a linear dependence of the peak amplitude on the logarithm of the filling-pulse duration (logarithmic capture law). This effect has been already observed for dislocations in plastically deformed $\mathrm{Si}$ [8, 13] or GaAs [12] as well as in lattice-mismatched InGaAs/GaAs heterostructures grown by metalorganic vapor phase epitaxy (MOVPE) [14] and MBE [1]. On the other hand, the isolated point defects or impurities exhibit exponential capture kinetics (exponential capture law).

Furthermore, it has been recently shown by Schröter et al. [5] that electronic states associated with spatially extended defects can be classified as band-like or localized ones when the rate $R_{\mathrm{i}}$ at which the states reach their internal electron equilibrium is taken into account. The internal rate $R_{\mathrm{i}}$, compared with the rates $R_{\mathrm{e}}$ (carrier emission rate) and $R_{\mathrm{c}}$ (capture rate), allows us to distinguish the localized states $\left(R_{\mathrm{i}} \ll R_{\mathrm{e}}, R_{\mathrm{c}}\right)$ from the band-like ones $\left(R_{\mathrm{i}} \gg R_{\mathrm{e}}, R_{\mathrm{c}}\right)$. It is also possible to classify them by means of DLTS. The criteria concerning the variation of the DLTS line with filling pulse duration have been derived in order to distinguish between these both types of states. Namely, for localized states, the DLTS line maximum remains constant when the filling pulse duration is changed, whereas the line amplitude exhibits a linear dependence on the logarithm of the filling time. On the contrary, in the case of bandlike states, variation of the filling pulse time results in a broadened DLTS line and its maximum shifts towards lower temperatures when the filling pulse time increases [5].

The features of DLTS line shape and its response to the filling pulse have been employed in our studies of distinguishing between isolated point defects and extended defects, such as dislocations.

\section{Experiment}

The InGaAs/GaAs heterostructures were grown by atmospheric pressure MOVPE system, fitted with AIX-200 R\&D horizontal reactor made by AIXTRON. The organometalic group III sources were TMGa (trimethylgallium) and TMIn (trimethylindium) and were transported by passing $\mathrm{H}_{2}$ through bubblers. $\mathrm{AsH}_{3}$ was used as the arsenic source reactant. The epitaxial layers were grown on semi-insulating (001)-oriented GaAs substrate at $670^{\circ} \mathrm{C}$ growth temperature. They consist of $n$-type, unintentionally doped at about $5 \times 10^{16} \mathrm{~cm}^{-3}, \mathrm{In}_{x} \mathrm{Ga}_{1-x}$ As layers with the In content of $4.2 \%$ resulting in the lattice-mismatch of $0.3 \%$ between GaAs and the ternary compound. The InGaAs layer thickness, of about 190 $\mathrm{nm}$, exceeded slightly the critical layer thickness, so the structure was partially relaxed. Prior to the growth of InGaAs layer, a $500 \mathrm{~nm}$ thick $n$-GaAs buffer was deposited. The composition and thickness of epitaxial layer were determined using X-ray diffraction (XRD) and electrochemical capacitance--voltage (ECV) profiling methods. In order to perform DLTS measurements, Schottky diodes were prepared by electron beam evaporation of $0.78 \mathrm{~mm}^{2}$ circular $\mathrm{Ti} / \mathrm{Pd} / \mathrm{Au}$ contacts, using 
standard lift-off, on a front side of the sample, and annealing was carried out at $250^{\circ} \mathrm{C}$ for $30 \mathrm{~min}$ in $\mathrm{N}_{2}$. Prior to the Schottky contact formation, the ring-shaped ohmic contacts were obtained by evaporating and annealing $\left(450^{\circ} \mathrm{C}, 3\right.$ min in $\left.\mathrm{N}_{2}\right)$ $\mathrm{Au} / \mathrm{Ge} / \mathrm{Ni} / \mathrm{Au}$ alloy. The quality of the Schottky barriers was checked up by the $I-V$ and $C-V$ measurements, showing good rectifying characteristics.

All the DLTS measurements were carried out within the $150 \div 410 \mathrm{~K}$ temperature range using a DLS-82E spectrometer. This system is based on $1 \mathrm{MHz}$ capacitance bridge meter and a lock-in integrator. The double correlation DLTS method (DDLTS) with two filling pulses, $U_{1}$ and $U_{2}$, of different heights $(-0.5 \mathrm{~V}$ and $-1.0 \mathrm{~V}$, respectively) and the same filling time was used. The value of reverse voltage $U_{\mathrm{R}}$ was kept constant, equal to $-3.0 \mathrm{~V}$. Laplace DLTS measurements were performed with the help of a digital oscilloscope Tektronix TDS1000 and software implemented in MATLAB environment, within the $310 \div 370 \mathrm{~K}$ temperature range. The signal obtained from the oscilloscope was filtered using 10th order Daubechies wavelet filter. In our studies, we used the Laplace transform algorithm introduced by Provencher [15], and adapted to the DLTS measurements by Kancleris and Matulis [16].

\section{Results discussion and conclusions \\ 4.1.Lock-in DLTS measurements}

The DDLTS measurements revealed the presence of five dominant deep electron traps labeled EG1-EG5. DLTS temperature scans for different filling-pulse times and the same lock-in frequency are shown in Fig 1. All measured spectra are symmetrically or asymmetrically broadened. In accordance with the previous information, the non-exponential transients causing the DTLS broadening can be related to many factors. In this paper, the anomalous DLTS line shape has been expected to be connected with stress induced misfit and threading dislocations.

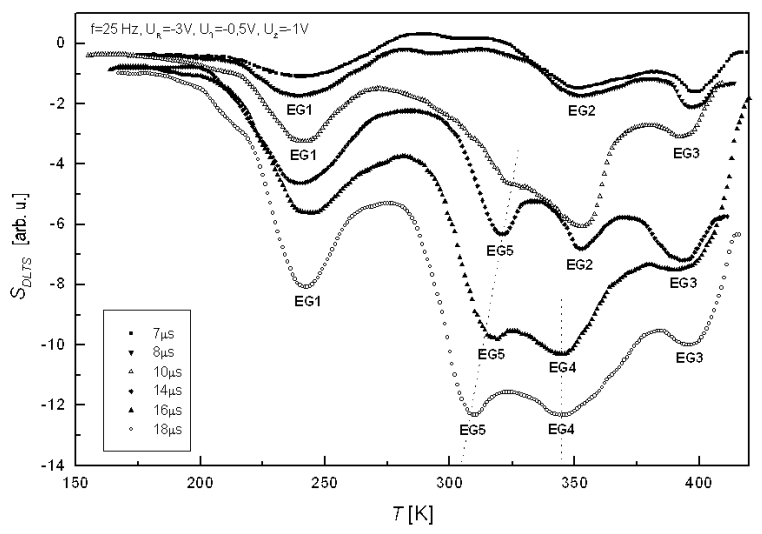

Fig. 1. DLTS spectra of the $\operatorname{In}_{0.042} \mathrm{Ga}_{0.958} \mathrm{As} / \mathrm{GaAs}$ system for different filling-pulse times. 
Temperature scans for several lock-in frequencies made possible to construct the Arrhenius plots for each DLTS signal peak (Fig 2). It has been shown [7] that the thermal emission rate $\left(e_{\mathrm{n}}\right)$, and the mean value of the thermal activation energy $\left(E_{\mathrm{a}}\right)$ are still directly obtainable although DLTS peaks are distorted. The application of the standard least-squares fitting procedure yielded the values of activation energies $E_{\mathrm{a}}$ and capture cross-sections $\sigma_{\mathrm{n}}$.

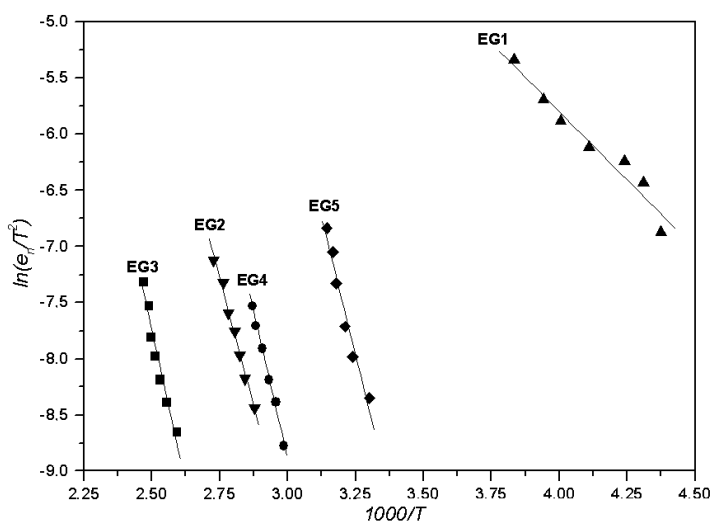

Fig. 2. Arrhenius plots for all electron traps, observed by DDLTS.

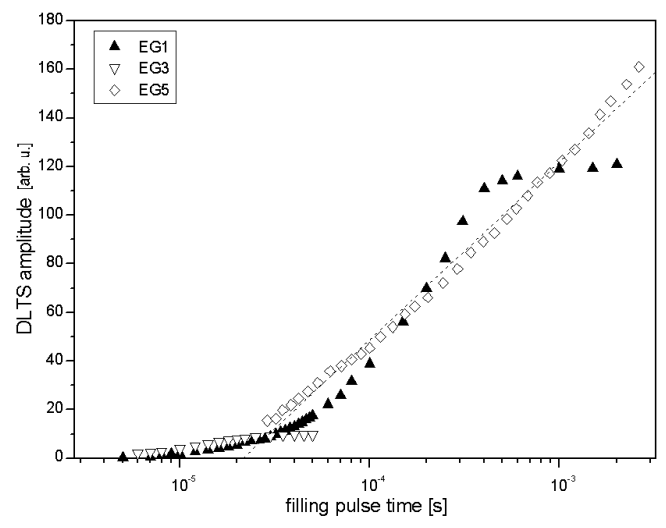

Fig. 3. Amplitudes of the DLTS peaks of EG1, EG3 and EG5 traps as a function of the filling-pulse time.

Basing on the capture kinetics and depth profile measurements the origin of the traps can be suggested. The analysis of EG1 and EG3 peaks allowed us to affirm that their broadening is due to the existence of the adjacent EG5 and EG4 peaks, respectively. Moreover, the amplitudes of these traps show a distinct saturation for long filling times (Fig. 3), typical of point defects. Besides, the 


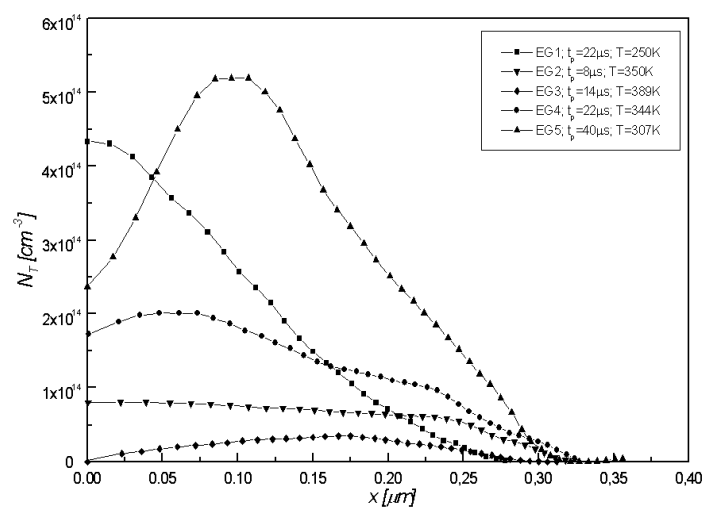

Fig. 4. DDLTS depth profiles for all observed electron traps.

maximum concentration of the EG1 trap is near the surface (Fig. 4). The concentrations of the EG3 trap remain nearly constant throughout the epilayer as is expected for the isolated point defect. From the emission characteristics and obtained parameters, the EG2 trap was identified as EL2, the dominant deep level defect in GaAs crystals.

The line amplitude of the EG5 trap exhibits linear dependence on the logarithm of a filling time (Fig. 3). This is the principal argument for attributing this trap to a dislocation $[12,13]$. Due to the overlapping with the EG4 line we were not able to exclude whether this plot is a sum of EG5 and EG4 lines. Therefore, the EG4 line can be correlated with dislocations. From the behaviour of EG4 and EG5 lines, we concluded that the DLTS-line maximum of the EG5 trap shifts towards lower temperatures for increasing filling time, whereas the EG4 line remains constant (Fig. 1). Such an effect allowed us to assign EG5 to band-like states and EG4 to localized states [5], but the origin of the latter trap was still unknown.

\subsection{Laplace DLTS measurements}

In order to determine precisely the parameters of the overlapping DLTS lines of EG2, EG4, and EG5 peaks, high resolution Laplace DLTS method has been used. The LDLTS studies carried out within the $310370 \mathrm{~K}$ temperature range revealed the presence of three deep electron traps, as it was expected. Laplace DLTS emission rate spectra (Fig 5) taken for several temperatures made possible to construct the Arrhenius plots for each of the peak (Fig 6). A linear regression fitting procedure yields the values of activation energies and capture cross-sections.

The parameters for both EG2 and EG5 traps were in accordance with the previous results, unlikely to the EG4 trap where the parameters were completely different. This discrepancy can be caused by the overlapping with the EG5 and EG2 peaks (Fig.1). A comparison of the obtained emission characteristics of the EG4 line with the data from the literature shows an excellent agreement with the 


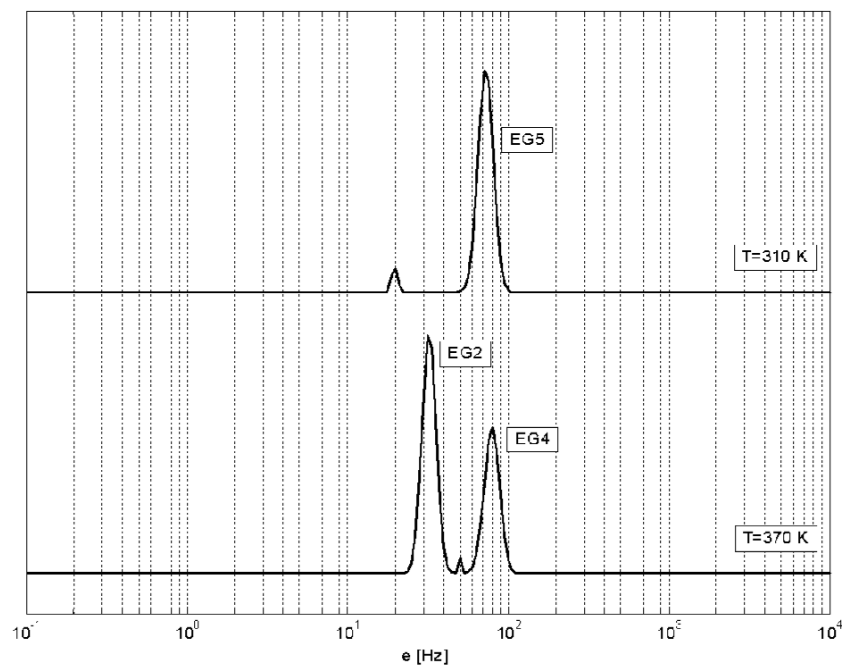

Fig. 5. LDLTS emission rate spectra.

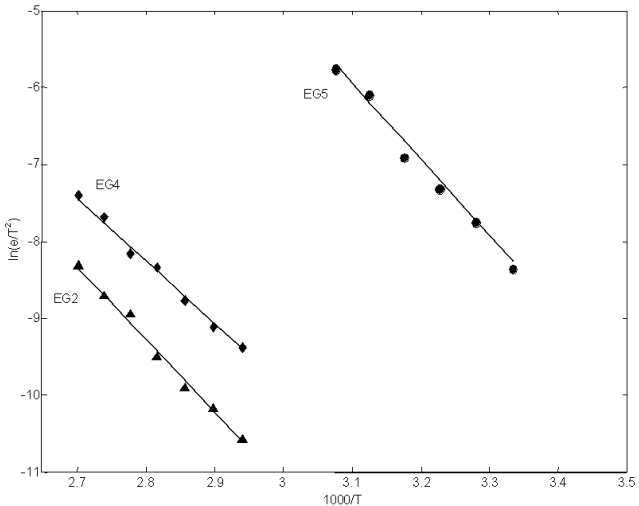

Fig. 6. Arrhenius plots for peaks obtained with LDLTS method.

TABLE

Activation energies $\left(E_{\mathrm{a}}\right)$, capture cross-sections $\left(\sigma_{\mathrm{n}}\right)$ and identification of the traps.

\begin{tabular}{c|c|c|c|c|c}
\hline \hline \multirow{2}{*}{ Level } & \multicolumn{2}{|c|}{$E_{\mathrm{a}}[\mathrm{eV}]$} & \multicolumn{2}{|c|}{$\sigma_{\mathrm{n}}\left[\mathrm{cm}^{2}\right]$} & \multirow{2}{*}{ Identification } \\
\cline { 2 - 5 } & DDLTS & LDLTS & DDLTS & LDLTS & \\
\hline EG1 & 0.21 & - & $2.6 \times 10^{-19}$ & - & point defect \\
EG2 & 0.79 & 0.81 & $3.19 \times 10^{-13}$ & $2.01 \times 10^{-13}$ & native point defect (EL2) \\
EG3 & 0.94 & - & $1.64 \times 10^{-12}$ & - & point defect \\
EG4 & 0.90 & 0.70 & $2.77 \times 10^{-11}$ & $1.56 \times 10^{-14}$ & threading dislocation (ED1) \\
EG5 & 0.85 & 0.86 & $1.4 \times 10^{-10}$ & $5.51 \times 10^{-10}$ & misfit dislocation
\end{tabular}


ED1 level attributed to the core states of dislocation by Wosiński [12]. Basing on these findings and the analysis of concentration depth profile (Fig.4), we concluded that the EG4 trap can be correlated with a threading dislocation. The EG5 trap can be attributed to a misfit dislocation near to the interface. All the deep level parameters determined for each trap by means of DDLTS and LDLTS methods, are summarized in Table.

\section{References}

[1] O. Yastrubchak, T. Wosiński, A. Mąkosa, T. Figielski, A.L. Tóth, Physica B 308-310, 757 (2001).

[2] D.V. Lang, J. Appl. Phys. 45, 3023 (1974).

[3] T. Figielski, Phys. Status Solidi A 121, 187 (1990).

[4] L. Dobaczewski, P. Kaczor, I.D. Hawkins, A.R. Peaker, J. Appl. Phys. 76, 194 (1994).

[5] W. Schröter, J. Kronewitz, U. Gnauert, F. Riedel, M. Seibt, Phys. Rev. B 52, 13726 (1995).

[6] L.C. Kimerling, J.R. Patel, Appl. Phys. Lett. 34, 73 (1979).

[7] P. Omling, L. Samuelson, H. G. Grimmeiss, J. Appl. Phys. 54, 5117 (1983).

[8] P. Omling, E. R. Weber, L. Montelius, H. Alexander, J. Michel, Phys. Rev. B 32, 6571 (1985).

[9] D. Stievenard, M. Lannoo, J.C. Bourgoin, Solid-State Electron. 28, 485 (1985).

[10] D. Pons, J. Appl. Phys. 55, 3644 (1984).

[11] A. Ito, Y. Tokuda, Solid-State Electron. 46, 1307 (2002).

[12] T. Wosiński, J. Appl. Phys. 65, 1566 (1989).

[13] T. Figielski, Solid-State Electron. 21, 1403 (1978).

[14] L. Panepinto, U. Zeimer, W. Seifert, M. Seibt, F. Bugge, M. Weyers, W. Schröter, Mater. Sci. Eng. B 42, 77 (1996).

[15] S.W. Provencher, Comp. Phys. Commun. 27, 213 (1982).

[16] Z. Kancleris, A. Matulis, Lithuanian J. Phys. 37, 475 (1997). 